\title{
Identificación de Parámetros Borrosos para el Control de Suspensión Activa mediante Enjambre de Partículas
}

\author{
Isabel Elena Herrera, Anthony Mandow y Alfonso García-Cerezo \\ Universidad de Málaga, Andalucía Tech, Dpto. Ingeniería de Sistemas y Automática \\ amandow@uma.es
}

\begin{abstract}
Resumen
Este artículo aborda la identificación de parámetros borrosos mediante técnicas de optimización de enjambre de partículas (PSO) y su aplicación al control de un sistema de suspensión activa. En particular, se adopta un controlador de tipo Takagi-Sugeno de orden cero con Partición Difusa Estándar de sus antecedentes. A diferencia de trabajos previos, donde el aprendizaje se limitaba a los parámetros de escala del control, el método propuesto permite la optimización de los conjuntos borrosos de los antecedentes. La metodología propuesta se ha experimentado con éxito sobre un sistema físico de un cuarto de vehículo.
\end{abstract}

Palabras clave: Control borroso, enjambre de partículas, suspensión activa, algoritmos evolutivos.

\section{Introducción}

Los sistemas de suspensión activa y semi-activa representan un problema de control relevante en el ámbito de la automoción. Asimismo, estos sistemas presentan dificultades inherentes, como su no linealidad y el conflicto entre confort y respuesta en conducción, que los convierten en una aplicación interesante para la investigación de nuevas estrategias para el diseño de controladores [4][8][17].

El control basado en lógica borrosa es un tipo de control inteligente que permite la posibilidad de expresar conocimiento heurístico en forma de reglas a la vez que ofrece características apropiadas para sistemas complejos [1]. Sin embargo, el ajuste de los controladores borrosos no es trivial, por lo que resultan apropiadas técnicas de optimización metaheurísticas como el recocido simulado [5] o los algoritmos genéticos [11].

Otro de estos métodos es la optimización por enjam- bre de partículas (PSO, del inglés Particle Swarm Optimization), una estrategia de optimización bioinspirada que simula el comportamiento social de grupos de seres vivos en la naturaleza, como enjambres de abejas o bancos de peces [9]. PSO puede proporcionar buenas características de convergencia y tiempo de cómputo respecto a otros métodos estocásticos [14] y se ha aplicado a diversos problemas en la ingeniería, incluyendo la sintonización de controladores PID para sistemas de suspensión [10].

El algoritmo PSO también se ha aplicado con sistemas borrosos, como en el ajuste de un control borroso definido mediante tan solo tres parámetros, aprovechando particiones borrosas simétricas y similares para las distintas variables [12]. En el ámbito de la suspensión activa, PSO ha servido para optimizar un modelo de predicción utilizado en combinación con el controlador borroso de la suspensión [15]. Asimismo, se ha empleado para ajustar los parámetros de escala en reguladores borrosos con funciones de pertenencia predefinidas [6][14].

Este trabajo propone una aplicación novedosa de PSO para la sintonización de reguladores borrosos, en la que se optimizan los parámetros de las funciones de pertenencia. Con este fin, se adopta un controlador de tipo Takagi-Sugeno de orden cero con partición difusa estándar [18] de sus antecedentes. El método propuesto se ha aplicado al control de un sistema de suspensión de cuarto de vehículo. Los resultados experimentales, realizados sobre una planta física, ofrecen una mejora del rendimiento respecto al control borroso con particiones fijas.

El resto del artículo se organiza de la siguiente manera. La siguiente sección repasa la la notación y definiciones de las particiones estándar. La sección 3 presenta el método de identificación de parámetros mediante PSO. En la sección 4 se presenta la aplicación al control de suspensión activa. A continuación, la sección 5 ofrece resultados experimentales. Para cerrar el artículo se presentan las conclusiones. 


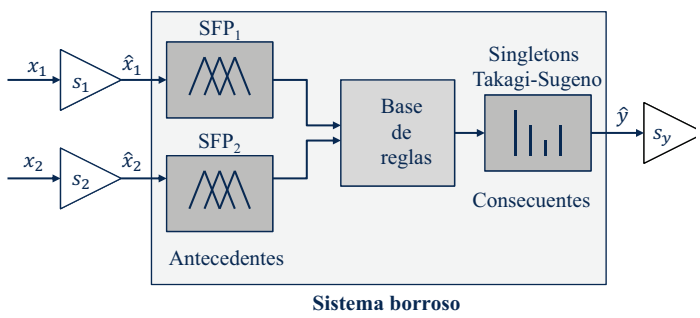

Figura 1: Estructura de un sistema borrosı Takagi-Sugeno con dos antecedentes SPF :

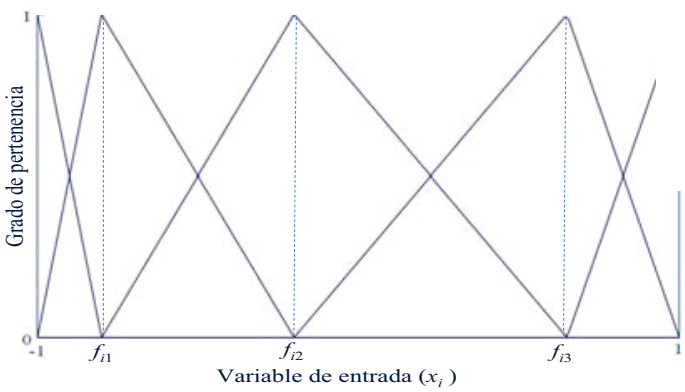

Figura 2: Ilustración de conjuntos triangulares en una partición SFP para $M=5$.

\section{Takagi-Sugeno con Particiones Estándar}

En este trabajo se propone el ajuste de los parámetros de los antecedentes para reglas borrosas con inferencia Takagi-Sugeno [16], como se ilustra en la Figura 1. Para la definición de los antecedentes se adopta la Partición Difusa Estándar (SPF, del inglés Standard Fuzzy Partition). Asimismo, se supone que los universos de discurso de las entradas y la salida están normalizados en el intervalo $[-1,1]$, lo cual implica factores de escala fuera del sistema borroso. Se supondrá que el sistema tiene $N_{v}$ variables de entrada $\left(x_{1}, \ldots, x_{i}, \ldots x_{N_{v}}\right)$ con $M$ particiones borrosas cada una.

En una partición SPF, la j-ésima Función de Pertenencia (MF, del inglés Membership Function) $\mathbf{F}_{j}$ definida para una variable dada $x_{i}$ en el universo de discurso $U$ es convexa, está normalizada y solo se solapa con sus MF vecinas:

$$
\forall u \in U, \quad \sum_{\forall i} \mathbf{F}_{j}(u)=1 .
$$

En el caso de MF triangulares, $\mathbf{F}_{j}$ se define mediante tres puntos, de los cuales el central es valor modal

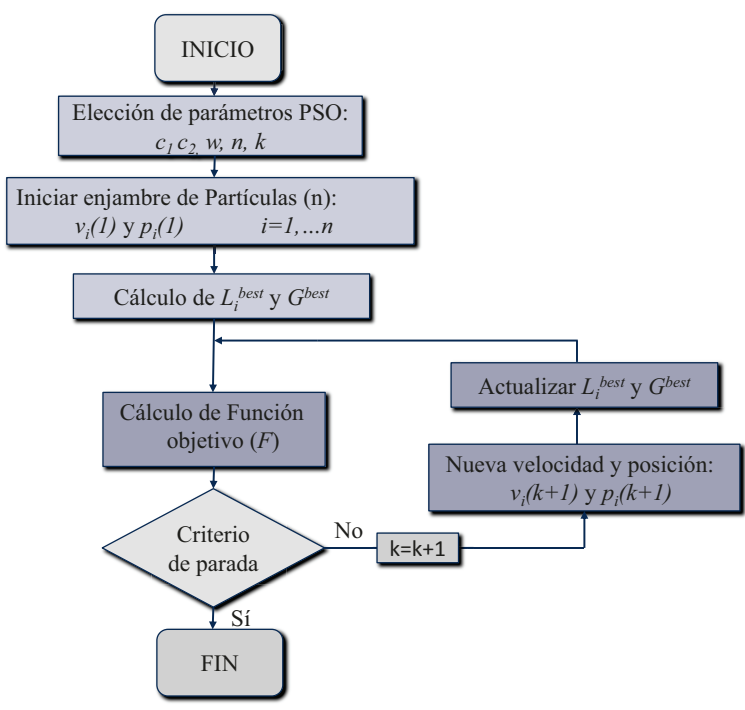

Figura 3: Diagrama de flujo del algoritmo PSO.

$f_{j}$ (véase un ejemplo en la Figura 2). La adopción de particiones SPF permite reducir el número total de parámetros, ya que cada conjunto triangular comparte valores con sus MF vecinas [18]. Así, la estructura de los $M$ conjuntos borrosos triangulares para una variable de entrada $x_{i}$ queda definida por un vector de valores modales de las MFs $\mathbf{f}_{i}=\left[f_{i 1} \ldots f_{i j} \ldots f_{i(M-2)}\right]$. Nótese que los valores $f_{i 0} \mathrm{y}$ $f_{i(M-1)}$ no se han incluido porque corresponden a los extremos del universo de discurso $[-1,1]$.

\section{Identificación de Parámetros Borrosos mediante PSO}

PSO permite optimizar una función objetivo en un espacio de búsqueda $N$-dimensional. Con este fin, se define un conjunto $n$ de partículas (i.e., soluciones candidatas) que evolucionan en las sucesivas iteraciones del algoritmo (ver Figura 3). Este método admite funciones objetivo no derivables, es relativamente sencillo de implementar, converge a soluciones razonables y su componente aleatorio sirve para evitar mínimos locales [9].

La evolución de la $i$-ésima partícula depende de su posición $\left(p_{i}\right)$ y velocidad $\left(v_{i}\right)$ en cada iteración $(k)$ :

$$
\begin{gathered}
v_{i}(k+1)=w v_{i}(k)+c r_{1}(k)\left(L_{i}^{\text {best }}-p_{i}(k)\right) \\
+c_{2} r_{2}(k)\left(G^{\text {best }}-p_{i}(k)\right) \\
p_{i}(k+1)=p_{i}(k)+v_{i}(k),
\end{gathered}
$$

donde $w$ es el parámetro de inercia del enjambre, $c_{1}$ es un componente cognitivo que atrae a las partí- 
culas hacia la mejor posición local, $c_{2}$ es un componente social que las alienta hacia la mejor posición global del enjambre, $L_{i}^{\text {best }}$ es la mejor posición hallada por la partícula $i, G^{b e s t}$ es la mejor posición global y $r_{1}$ y $r_{2}$ son números aleatorios entre 0 y 1 .

Para la identificación de parámetros borrosos de los antecedentes, una partícula queda definida por un vector $\left[\mathbf{f}_{1} \ldots \mathbf{f}_{N_{v}}\right]$, donde los valores modales de cada $\mathbf{f}_{i}$ se ordenan de menor a mayor. Esto corresponde a una dimensión del espacio de búsqueda:

$$
N=N_{v} \cdot(M-2) .
$$

\section{Control Borroso de Suspensión Activa}

La Figura 4 muestra el esquema de control borroso de suspensión activa de un cuarto de vehículo. En el sistema de suspensión, $z_{s}$ y $z_{u s}$ representan los respectivos desplazamientos de la masa suspendida y de la masa no suspendida ante una perturbación en la carretera $z_{r}$. Las características del sistema las definen los coeficientes de rigidez $\left(k_{s}\right.$ y $\left.k_{u s}\right)$ y de amortiguamiento ( $B_{s}$ y $\left.B_{u s}\right)$.

En cuanto al controlador borroso, su objetivo es mantener la aceleración de la masa suspendida $\left(A_{s}\right)$ lo más próxima a cero. Con este fin, se definen dos variables de entrada: $e$, que representa la desviación de $z_{s}$ respecto a $z_{r}, \mathrm{y} e_{r}$, que corresponde a la derivada de $e$. La salida del sistema borroso es la fuerza de actuación $F_{a}$. Todas las variables se encuentran normalizadas en $[-1,1]$ mediante factores de escala.

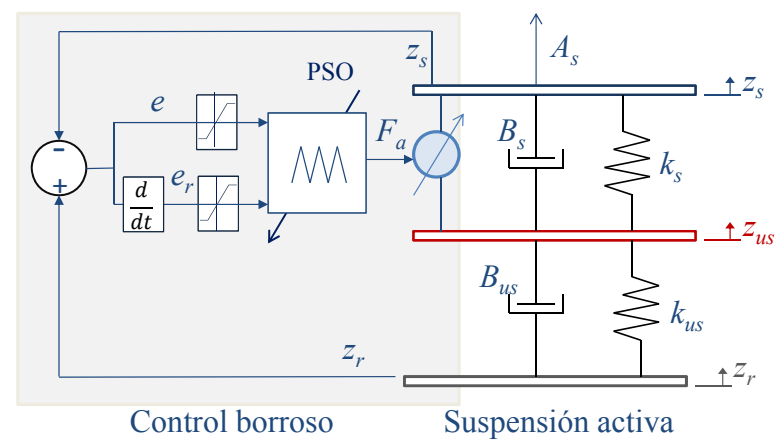

Figura 4: Esquema suspensión activa de un cuarto de vehículo mediante control borroso.
Cuadro 1: Reglas heurísticas de control para un sistema de suspensión activa.

\begin{tabular}{|c|c|c|c|c|c|c|c|c|}
\hline & \multicolumn{7}{|c|}{ error } \\
\hline & & $\mathrm{PB}$ & $\mathrm{PM}$ & PS & $\mathrm{ZE}$ & NS & $\mathrm{NM}$ & NB \\
\hline \multirow{7}{*}{$\begin{array}{l}\tilde{0} \\
\tilde{0} \\
\tilde{0} \\
\tilde{U} \\
\widetilde{W} \\
\widetilde{W} \\
\tilde{W}\end{array}$} & $\mathrm{~PB}$ & $\mathrm{~PB}$ & $\mathrm{~PB}$ & $\mathrm{~PB}$ & $\mathrm{~PB}$ & $\mathrm{PM}$ & $\mathrm{PS}$ & $\mathrm{ZE}$ \\
\hline & $\mathrm{PM}$ & $\mathrm{PB}$ & $\mathrm{PB}$ & $\mathrm{PB}$ & $\mathrm{PM}$ & PS & $\mathrm{ZE}$ & NS \\
\hline & PS & PB & $\mathrm{PB}$ & $\mathrm{PM}$ & PS & $\mathrm{ZE}$ & NS & $\mathrm{NM}$ \\
\hline & $\mathrm{ZE}$ & $\mathrm{PB}$ & $\mathrm{PM}$ & PS & $\mathrm{ZE}$ & NS & $\mathrm{NM}$ & $\mathrm{NB}$ \\
\hline & NS & $\mathrm{PM}$ & PS & $\mathrm{ZE}$ & NS & $\mathrm{NM}$ & NB & NB \\
\hline & $\mathrm{NM}$ & PS & $\mathrm{ZE}$ & NS & $\mathrm{NM}$ & $\mathrm{NB}$ & $\mathrm{NB}$ & $\mathrm{NB}$ \\
\hline & NB & $\mathrm{ZE}$ & NS & NM & NB & NB & NB & NB \\
\hline
\end{tabular}

da variable de entrada y siete términos lingüísticos para la variable de salida (i.e., siete valores singletons equidistantes en el caso de inferencia TakagiSugeno). Así, aplicando (4) el número de parámetros a optimizar es $N=10$, que corresponden a los valores modales de $P M, P S, Z E, N S, N M$ para las variables $e$ y $e_{r}$.

PSO se inicializa con $n$ partículas distribuidas aleatoriamente en el espacio de búsqueda. Los otros parámetros de la ecuación (2) se han hallado experimentalmente. A partir de valores plasmados en trabajos anteriores y realizando ensayos, se han elegido finalmente aquellos parámetros que reducen el tiempo de convergencia.

La función objetivo se ha definido así:

$$
F_{o b j}=\int E^{2}+z_{s}^{\max }
$$

donde $E$ es el error cuadrático medio y $z_{s}^{\max }$ es la máxima posición que ha presentado la masa suspendida del sistema.

\section{Resultados Experimentales}

\subsection{Planta Experimental}

La planta de suspensión activa Quanser es un modelo a escala que emula el comportamiento de un cuarto de vehículo (ver Figura 5). Está planta está compuesta por tres placas de acero: La placa superior, que incluye un acelerómetro, simula la masa suspendida del vehículo; la placa intermedia, la masa no suspendida y la placa inferior, la carretera. La posición de las masas suspendida y no suspendida se obtienen a partir de codificadores angulares. El sistema cuenta con dos motores: el primero sirve para provocar las perturbaciones de la carretera y el segundo es el actuador para la suspensión activa. 


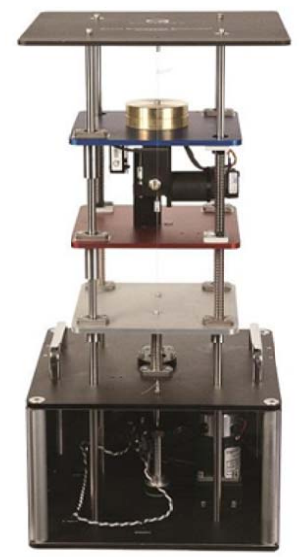

Figura 5: Sistema experimental de suspensión activa Quanser [13].

Cuadro 2: Parámetros del sistema de suspensión [13].

\begin{tabular}{lr} 
Parámetro & Valor \\
\hline \hline$K_{s}$ & $2000 \mathrm{~N} / \mathrm{m}$ \\
$B_{s}$ & $2500 \mathrm{Ns} / \mathrm{m}$ \\
$K_{u s}$ & $10 \mathrm{~N} / \mathrm{m}$ \\
$B_{u s}$ & $30 \mathrm{Ns} / \mathrm{m}$ \\
\hline
\end{tabular}

Cuadro 3: Parametrización del algoritmo PSO.

\begin{tabular}{lr} 
Parámetro & Valor \\
\hline \hline$n$ & 20 \\
$k_{\max }$ & 110 \\
$N$ & 10 \\
$w$ & 0.9 \\
$c_{2}$ & 2.2 \\
$c_{1}$ & 1.8 \\
\hline
\end{tabular}

La conexión con el ordenador de control se realiza mediante una tarjeta de adquisición de datos del propio fabricante. En el cuadro 2 se muestran los parámetros de la planta experimental.

\subsection{Experimentos}

En esta sección se presentan resultados experimentales de la aplicación del control borroso optimizado mediante PSO con la planta Quanser. Además, se comparan con el caso en el que únicamente se optimizan los parámetros de escala de entrada y salida [7], resultando en $s_{1}=93,4563, s_{2}=30,6043$ y $s_{y}=15,5411$. El método propuesto se ha aplicado sobre un sistema borroso normalizado aplicando esas mismas escalas.
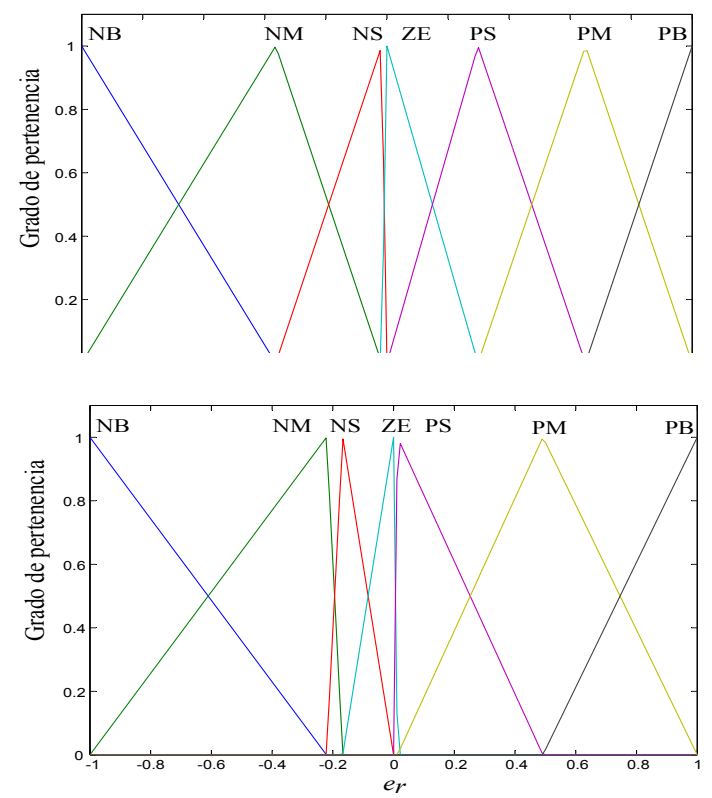

Figura 6: Particiones borrosas optimizadas para $e$ (arriba) y $e_{r}$ (abajo).

Para la optimización PSO, se ha realizado una simulación fuera de línea de cada solución candidata mediante un modelo Matlab/Simulink de la planta [13]. En el Cuadro 3 se muestran los parámetros usados para la implementación de PSO.

En este caso se evalúa la respuesta de los controladores frente a un bache en $t=2 s$ y un obstáculo en $t=4 \mathrm{~s}$. Estas perturbaciones se representan mediante un pulso cuadrado de $\pm 0,01 \mathrm{~m}$ con un periodo de $4 s$ y un ancho de pulso de $2 s$. También se analiza la respuesta del sistema frente a una entrada ruido aleatorio. Para estas entradas, las particiones difusas estándar proporcionadas por la simulación fuera de línea se muestran en la Figura 6 .

Para $z_{r}$ de tipo bache y obstáculo, se observa en la Figura 7 que el controlador propuesto en este trabajo ofrece una respuesta más suave que el controlador formado únicamente por parámetros de escala. Los valores de aceleración máxima y tiempo de establecimiento son menores.

El incremento del rendimiento en el controlador propuesto también se observa en la respuesta de la posición de la masa suspendida (ver Figura 8). Tanto el valor máximo de la posición de la masa suspendida como la amplitud de la sobreoscilación son menores en el controlador propuesto. Por otro lado, en la Figura 8 se aprecian diferencias el valor estacionario de la posición de la masa suspendida entre los 

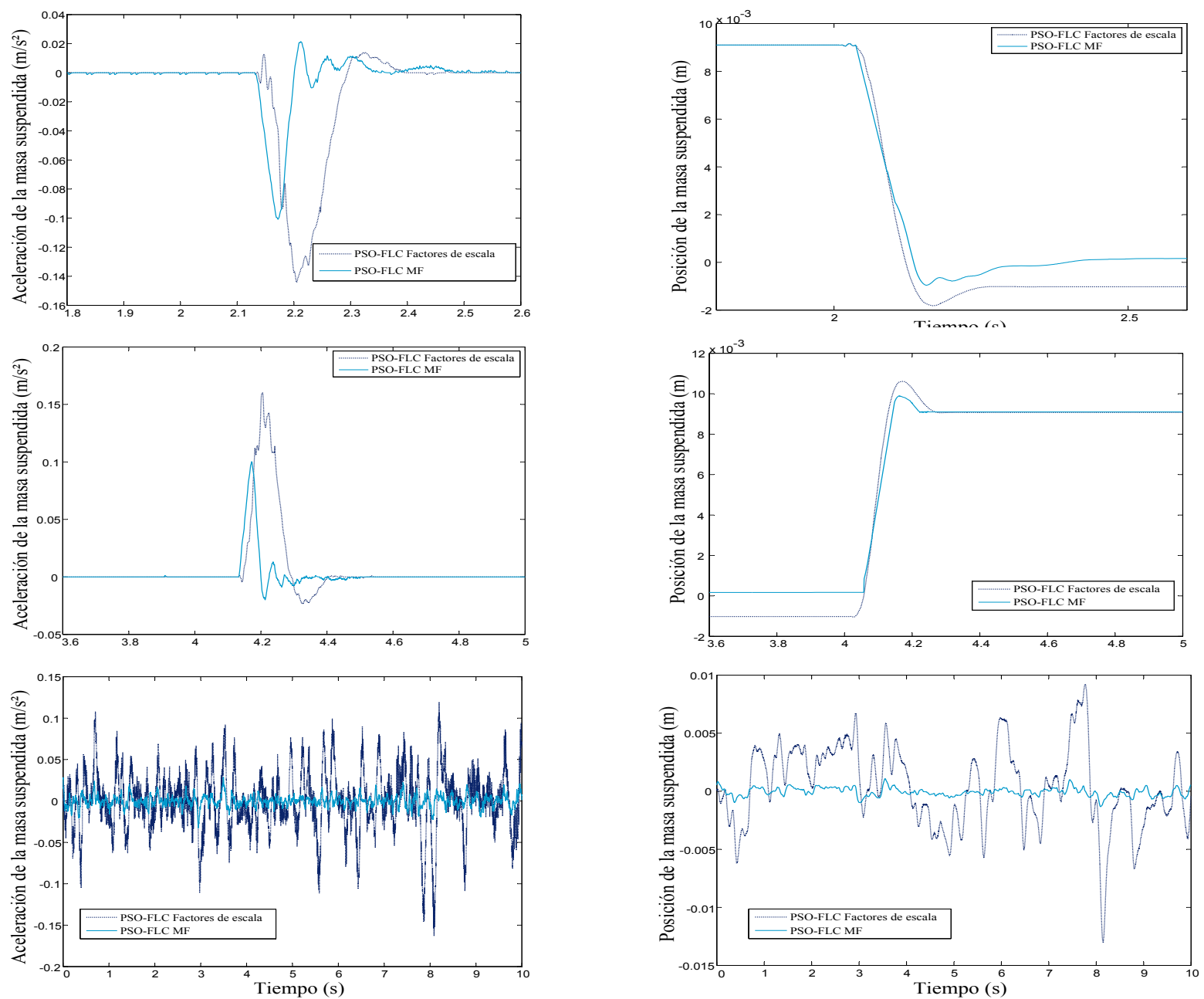

Figura 7: Respuesta temporal de la aceleración de la masa suspendida $A_{c}$ ante bache (arriba), obstáculo (centro) y ruido (abajo).

dos controladores borrosos. Este error se asocia a huelgos mecánicos de la planta experimental.

En el caso de $z_{r}$ de tipo ruido, los valores que proporciona el controlador propuesto tanto para la aceleración como para la posición de la masa suspendida son menos de un $20 \%$ de los valores que se obtienen con el controlador borroso en el que sólo se optimizan los factores de escala. Los resultados experimentales confirman que se consigue mejorar el comportamiento del controlador borroso al optimizar los parámetros de las MF.

\section{Conclusiones}

En este trabajo se ha propuesto la identificación de parámetros borrosos mediante técnicas de optimiza-

Figura 8: Respuesta temporal de la posición de la masa suspendida $z_{s}$ ante bache (arriba), obstáculo (centro) y ruido (abajo).

ción de enjambre de partículas (PSO) y se ha aplicado al control de un sistema de suspensión activa. El controlador considerado ha sido de tipo TakagiSugeno de orden cero con Partición Difusa Estándar de sus antecedentes, donde los parámetros que forman parte de las MF se han optimizado mediante PSO.

El controlador se ha comparado con trabajos previos, donde el aprendizaje se limitaba a los parámetros de escala del control. En particular, se ha evaluado la respuesta temporal ante tres tipos de perturbaciones típicas, obteniéndose una mejora notable en el rendimiento. En trabajos futuros se evaluará la posibilidad de utilizar PSO para la optimización de los consecuentes Takagi-sugeno. 


\section{Agradecimientos}

Este trabajo ha sido financiado parcialmente por los proyectos CICYT DPI 2011-22443 and DPI201565186-R.

\section{Referencias}

[1] E. Aranda-Escolástico, M. Guinaldo, M. Santos, and S. Dormido. Control of a chain pendulum: a fuzzy logic approach. International Journal of Computational Intelligence Systems, 9(2):281-295, 2016.

[2] Yi Chen. Skyhook surface sliding mode control on semi-active vehicle suspension system for ride comfort enhancement. Engineering, 1:2332,2009 .

[3] Seema Chopra, R. Mitra, and Vijay Kumar. A robust scheme for tuning of fuzzy PI type controller. In 3rd International IEEE Conference Intelligent Systems, pages 300-305. IEEE, September 2006.

[4] A. Colina, G. Lerma, I. Cabanes, and I. Iglesias. Modelling and control of a semi-active suspension system. Mechanisms and Machine Science, 17:25-32, 2014.

[5] R. E. Haber, R. Haber-Haber, A. Jiménez, and R. Galán. An optimal fuzzy control system in a network environment based on simulated annealing. an application to a drilling process. Applied Soft Computing Journal, 9(3):889-895, 2009 .

[6] J. Hurel, A. Mandow, and A. Garcia-Cerezo. Tuning a fuzzy controller by particle swarm optimization for an active suspension system. In IEEE Industrial Electronics Conference, pages 2524-2529, 2012.

[7] Jorge Hurel, Anthony Mandow, and Alfonso García-Cerezo. Nonlinear two-dimensional modeling of a McPherson suspension for kinematics and dynamics simulation. In The 12th IEEE International Workshop on Advanced Motion Control, pages 1-6, Sarajevo, Bosnia and Herzegovina, 2012.

[8] Jorge Hurel-Ezeta, Anthony Mandow, and Alfonso García-Cerezo. Los sistemas de suspensión activa y semiactiva: una revisión. Revista Iberoamericana de Automática e Informática Industrial, 10(2):121-132, 2013.
[9] R. C. Eberhart J. Kennedy. Particle swarm optimization. In IEEE International Conference on Neural Networks IV, page 1942-1948, 1995.

[10] H. Metered, A. Elsawaf, T. Vampola, and Z. Sika. Vibration control of mr-damped vehicle suspension system using pid controller tuned by particle swarm optimization. SAE International Journal of Passenger Cars - Mechanical Systems, 8(2):426-435, 2015.

[11] R.K. Pekgökgöz, M.A. Gürel, M. Bilgehan, and M. Kýsa. Active suspension of cars using fuzzy logic controller optimized by genetic algorithm. International Journal of Engineering and Applied Sciences, 2(4):27-37, 2010.

[12] R. E. Precup, R. C. David, A. I. Stinean, M. B. Radac, and E. M. Petriu. Adaptive hybrid particle swarm optimization-gravitational search algorithm for fuzzy controller tuning. In IEEE International Symposium on Innovations in Intelligent Systems and Applications, pages 1420, 2014.

[13] Quanser. User Manual, Active Suspension Experiment. 2012.

[14] K. Rajeswari and P. Lakshmi. PSO optimized fuzzy logic controller for active suspension system. In International Conference on Advances in Recent Technologies in Communication and Computing, pages 278-283, 2010.

[15] K. Rajeswari, S. Lavanya, and P. Lakshmi. Grey fuzzy sliding mode controller for vehicle suspension system. Control Engineering and Applied Informatics, 17(3):12-19, 2015.

[16] T. Takagi and M. Sugeno. Fuzzy identification of systems and its applications to modeling and control. IEEE Transactions on Systems, Man and Cybernetics, SMC-15(1):116-132, 1985.

[17] J.C. Tudón-Martínez, S. Varrier, R. MoralesMenendez, and O. Sename. Fault tolerant control in a semi-active automotive suspension [control tolerante a fallas en una suspensión automotriz semi-activa]. Revista Iberoamericana de Automática e Informática Industrial, 13(1):56-66, 2016.

[18] Z. H. Xiu and G. Ren. Stability analysis and systematic design of Takagi-Sugeno fuzzy control systems. Fuzzy Sets and Systems, 151(1):119-138, 2005. 